\title{
Interactive comment on "Sterol preservation in hypersaline microbial mats" by Yan Shen et al.
}

\section{Yan Shen et al.}

yshen@gwdg.de

Received and published: 8 August 2019

We sincerely thank referee \#1 (Rienk Smittenberg) for the constructive comments, which helped us to improve our manuscript. Below we list all the points raised by the reviewer (given between quotes), followed by our replies.

1. - "The use of TMCS in methanol to methylate fatty acids is rather uncommon. Please provide a reference where this method and its efficiency is described."

REPLY: We now provide a reference describing the use and efficiency of TMCS in methanol for the methylation of fatty acids by Poerschmann and Carlson (2006) in the materials and methods section.

Planned changes in manuscript: We will cite this reference in the revised version (chapter 2.3). 
2. - "How were the $13 \mathrm{C}$ contents of the sterols and FAs corrected for the added derivatizing group."

REPLY: We corrected 13C contents for the added derivatizing group following the method described by Goñi and Eglinton (1996). First, we analyzed Heneicosylic acid and Androstanol standards derivatized as methyl ester (ME-) and trimethylsilyl (TMS) ester by GC-C-IRMS to obtain the carbon isotopic value for the derivatizing groups, and after measuring our samples, we corrected the carbon isotope values of our derivatized lipids according to the equations provided in that study. This will be indicated in the materials and methods section.

Planned changes in manuscript: We will specify the method and add the reference (Goñi and Eglinton, 1996) in the revised version (chapter 2.6).

3. - "I wonder if pyrolysis GC-MS is the best way to assess if steroids make it into the 'kerogen' fraction of recent material. I am not a Py-GC-MS expert but pyrolysis at $560^{\circ} \mathrm{C}$ is rather high temperature where most organic molecules will break down to smaller pieces; any remaining intact compounds or larger fragments will be low in concentration. The fact that some hopanoids (fragments) may indicate that they are simply more abundant, while any remaining steroids could be below detection limit (i.e. below the background of the $213+215+217$ trace). Absence of evidence is not the same as evidence of absence...,"

REPLY: As demonstrated by for example Gelin et al. (1996), steroids are thermally stable at such high temperatures $\left(610^{\circ} \mathrm{C}\right.$ in Gelin et al., 1996) and Py-GC-MS is a suitable method to demonstrate their presence in immature kerogens. Another example demonstrating the value of Py-GC-MS for our objectives is the study by Kruge and Permanyer (2004), who applied Py-GC-MS at $600^{\circ} \mathrm{C}$ for evaluating steranes/sterenes as tracers for organic contamination in recent sediments. Finally, the results obtained from our reference material can be taken as proof that the applied method is suitable for the analysis of steroids bound to kerogen. These points will be indicated in the results and

Printer-friendly version

Discussion paper
Interactive comment 
discussion section.

We agree that "Absence of evidence is not the same as evidence of absence". In this regard, we will reword the discussion chapter and clarify that the kerogen-bound steroids are below our (individual) detection limit for Py-GC-MS. Regarding the definition of our detection limit, please see author's response below (5.).

Planned changes in manuscript: We will reword and add these points in the revised version (chapter 3.4 and 4.2 ).

4. - "... Information about the reference kerogen of the Green River shale is lacking (e.g. sample amount, or relative amount of hopanes-steranes in Green River bitumen) so this comparison does not tell very much."

REPLY: Our intention when displaying the reference run was to demonstrate the ability of the system to detect the compounds in question, and to indicate the exact retention times in the chromatograms. For that purpose we used about $0.5 \mathrm{mg}$ of kerogen purified from the Eocene Green River oil shale (Eastern Utah, White River Mine, BLM Oil Shale Research, Development, and Demonstration Lease UTU-84087).

Planned changes in manuscript: We will add detailed information about the reference material to the revised version (chapter 3.4; supplementary information).

5. - "More critically, Blumenberg (2015) could report hopane/sterane ratios for the same mat, which means steranes were present throughout - although they did appear to decrease with depth (at least compared to the hopanes). I would have the same critique to the already published paper about Lake 22 (Shen 2018) when it concerns the use of Py-GC-MS to investigate the presence of steranes in the "proto-kerogen."

REPLY: It is generally difficult to provide sound quantitative data with Py-GC-MS. An Printer-friendly version estimate for the detection limit of our system can be derived from our analysis of the samples together with an internal standard (n-eicosane D42, $120 \mathrm{ng}$ ) that was routinely added to check the performance of the chromatographic system. When comparing our

Discussion paper

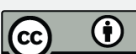


steroid peaks to this standard (and neglecting slightly different response factors), the amount of analyte should be about $1 \mathrm{ng}$ (absolute amount) to obtain a reliable mass spectrum that allows for identification. We admit that this is certainly higher than the detection limit of the HyPy-approach (Blumenberg et al. 2015), that includes chromatographic isolation, catalytic hydrogenation and concentration of the target analytes. This might partly explain the lack of steroid signals in our chromatograms (see also 6.).

Interactive

To clarify how steroids can be detected by our Py-GC-MS system, we have added below a screenshot (Figure 1) showing Py-GC-MS chromatograms (TICs) of the original microbial mat (Layer 2; upper chromatogram), and its extraction residue ('kerogen'; lower chromatogram). Sterenes and hopenes are abundant in the original mat sample, but their concentrations are below detection limit in the kerogen. Mass spectra for some compounds from the upper chromatogram are also given, representing a C27 sterene (1A), a C27 hopene (2A), and a C28 sterene (3A). Compound $3 A$ is just identifiable from the TIC (though some coelution is evident in the mass spectrum) and it was therefore used to define our (conservative) detection limit of $\sim 1 \mathrm{ng}$ (absolute amount) for kerogen-bound steroids.

Planned changes in manuscript: We will detail our detection limit, and clarify that our pyrolysis results cannot exclude the presence of small amounts of steroids in the kerogen fractions (abstract; chapter 3.4 and 4.2).

6. - "The paper cannot really be read without also consulting a more comprehensive hypy-GC-MS- biomarker analysis published by Blumenberg (2015), who finds hopane/sterane ratios of 20-100- thus no surprise there are no sterols found by PyGC-MS while hopanes do show a trace."

REPLY: As detailed above (5.), we agree that small amounts of steroids might not be detected by Py-GC-MS due to our detection limit.

Planned changes in manuscript: We will provide an improved discussion including a more detailed comparison with the results from Blumenberg et al. (2015), and a

Printer-friendly version

Discussion paper 
discussion of the detection limit (chapter 4.2).

7. - "Why did the authors not measure (or present) free hopanoids: Or for that matter a more comprehensive biomarker study (i.e. a free extractable lipid version of the Blumenberg paper). This would give the paper much more body, constraining it to only steroids feels very limited. I strongly recommend expanding the paper in this way."

REPLY: We agree that hopanoids may provide additional information, but our study was aimed at the fate of steroids. Whereas our GC-MS based approach is able to assess the whole range of steroids, it can only reveal some diagenetic products of the original polyfunctionalized hopanoids, e.g. hopanes/hopenes, and hopanols. Providing a comprehensive picture on the hopanoids would also require the additional analysis of polyfunctionalized hopanoids, and thus, the use of further techniques such as LC-MS, which we feel is beyond the scope of this study.

8. - "The $14 \mathrm{C}$ dating of carbonates on a coral atoll has a large risk of a reservoir effect (the coral is likely from the mid-Holocene sea level high stand thus several 1000 years old). Indeed a 14C age of just -239 years indicates a fraction modern of just over 1 , i.e. a mixture of post-bomb atmospheric $\mathrm{CO} 2$ and an ancient source. The downcore increase in age does make sense, but one cannot assign any exact ages to the mat material - for this, one needs to date plant macrofossils. I realize the results were published already by Blumenberg et al but they can only be interpreted as deeper=older."

REPLY: Since we consider the exact ages of these layers irrelevant for our purposes, we will follow the advice of the reviewer and not expand on the $14 \mathrm{C}$ data published by Blumenberg et al. (2015).

Planned changes in manuscript: We will remove the detailed age information (Figure 1 and chapter 3.1).

9. - "When looking at the depth profiles of the sterols, I do not see a clear decrease with depth, except the large difference between the surface layer and layers below the

Printer-friendly version

Discussion paper
Interactive comment 
phototrophic active part. Layer 5 and layer 2 have the same concentration per $\mathrm{g}$ TOC, and layer 3 and 6 the same expressed per g dry mat."

REPLY: In the manuscript, we refer to the same "large difference" between the surface and the deeper layers, as noted by the reviewer (e.g. P7, line 7-11). We did not mean that there was a gradual decrease with depth.

Planned changes in manuscript: The respective text passages will be reworded to clarify this (chapter 3.3).

10. - "Comparison with Lake 22 (Shen 2018): What is different between the two lakes is a halite-gypsum crust on top of Lake 22 - which must impair oxygen flux to the upper layer. Yet, Lake 22 shows considerably higher steroid concentrations than this Lake 2. This may explain the absence of higher sterol abundances in the upper layer (i.e. absence of eukaryotic sterol-producing photosynthetic organisms in Lake 22 but rather a 'fossil' signal starting already in layer 1 below the halite crust. The higher sterol conc. in Lake 22 may simply be a higher contribution from terrestrial vegetation, but as the authors state it can also be a lower degradation because of ultrahigh salinity. Coprostanol found in Lake 22 could be derived from the abundant land crabs on Kiritimati, which live around the lakes and eat the local vegetation (and each other - personal observation in 2005)."

REPLY: We agree that the halite-gypsum crust on top of the Lake 22 mat may have impaired oxygen flux to the upper layer, thereby reducing the production of sterols in the upper part of the Lake 22 mat. On the other hand, we do not consider the higher sterol concentrations in Lake 22 as being caused by a higher terrestrial input. This is demonstrated by the distributions of sterol pseudohomologues (high in C27) as well as the similar sterol concentrations in the top layers of both mats $(102 \mu \mathrm{g} / \mathrm{g}$ Corg range, see chapter 4.3, P10, line 26-27). As discussed in the manuscript, we consider hypersalinity, combined with periods of subaerial exposure, as more important factors on the degradation rates, and regard this a more likely explanation why steroids showed

Printer-friendly version

Discussion paper 
no overall systematic decrease throughout the mat profile in Lake 22.

Planned changes in manuscript: A discussion on the potential effect of a halite-gypsum crust on top of Lake 22 mat will be added to the revised version (chapter 4.3).

11. - "I also agree that the data confirm the hypothesis that microbial mats do not preserve original photosynthetic lipids from the upper very well, and that this signal is overprinted by heterotrophic organisms. However, marine or lake sediments do have the same' problem': organic matter degradation on heterotrophy within (anoxic) sediments, i.e. a diagenetic overprint. That said, these are not really very new insights, the added value compared to the Blumenberg 2015 and Shen 2018 papers is marginal."

REPLY: In the current work, we investigated a microbial mat from a lake with completely different environmental conditions as compared to the 2018 publication with respect to salinity and water depth. In addition, the microbial mat studied in 2018 probably experienced periods of subaerial exposure, which is not observed for the currently studied microbial mat. Further, unlike it has been done in the 2018 paper and in Blumenberg et al. (2015), we explicitly analyzed steroid distributions in freely extractable vs. carbonate-bound lipid fractions to test whether calcification within the microbial mat may function as a preservation mechanism for these biomarkers.

Planned changes in manuscript: We will reword and put more emphasis on the novel insights that our new work provided as compared to Blumenberg et al. (2015) and Shen et al. (2018) (abstract, chapter 4.3).

12. - "I do not agree with the conclusion that steroids are not preserved, in my opinion the authors have not used the right method to investigate this. Blumenberg (2015) found steranes after hypy."

REPLY: The reviewer is correct in that it cannot be concluded that steroids are not preserved in microbial mats, but we did not claim this in our manuscript. For the Lake 2 mat we demonstrate how sterols experienced major degradation that suppressed the

Printer-friendly version

Discussion paper 
primary ecological signal. In contrast, sterols in the Lake 22 mat (Shen et al., 2018) experienced major microbial transformation which largely preserved their molecular integrity. Taken together, these findings highlight that sterols may have contrasting preservation pathways in microbial mats, and that preservation may be much better in mats experiencing higher salinities and/or more desiccated conditions. This is certainly a relevant and novel insight revealed by this study.

Planned changes in manuscript: We will put more emphasis on the novel findings and sharpen the respective text passages in abstract, discussion, and conclusions sections (chapters 4.2, 4.3 and 5).

\section{References cited in the reply:}

Blumenberg M., Thiel V. and Reitner J. (2015). Organic matter preservation in the carbonate matrix of a recent microbial mat - Is there a 'mat seal effect'? Organic Geochemistry 87, 25-34.

Gelin F., Sinninghe Damsté J. S., Harrison W. N., Reiss C., Maxwell J. R. and De Leeuw J. W. (1996) Variations in origin and composition of kerogen constituents as revealed by analytical pyrolysis of immature kerogens before and after desulphnrization. Organic Geochemistry 24, 705-714.

Goñi M. A. and Eglinton T. I. (1996) Stable carbon isotopic analyses of lignin-derived $\mathrm{CuO}$ oxidation products by isotope ratio monitoring-gas chromatography-mass spectrometry (irm-GC-MS). Organic Geochemistry 24, 601-615.

Kruge M. A. and Permanyer A. (2004) Application of pyrolysis-GC/MS for rapid assessment of organic contamination in sediments from Barcelona harbor. Organic Geochemistry 35, 1395-1408. based on "in-cell fractionation" using sequential pressurized liquid extraction. Journal of chromatography. A 1127, 18-25. 
Shen Y., Thiel V., Duda J.-P. and Reitner J. (2018). Tracing the fate of steroids through a hypersaline microbial mat (Kiritimati, Kiribati/Central Pacific). Geobiology 16, 307-318.

Please also note the supplement to this comment:

https://www.biogeosciences-discuss.net/bg-2019-124/bg-2019-124-AC1-

supplement.pdf

Interactive comment on Biogeosciences Discuss., https://doi.org/10.5194/bg-2019-124, 2019. 


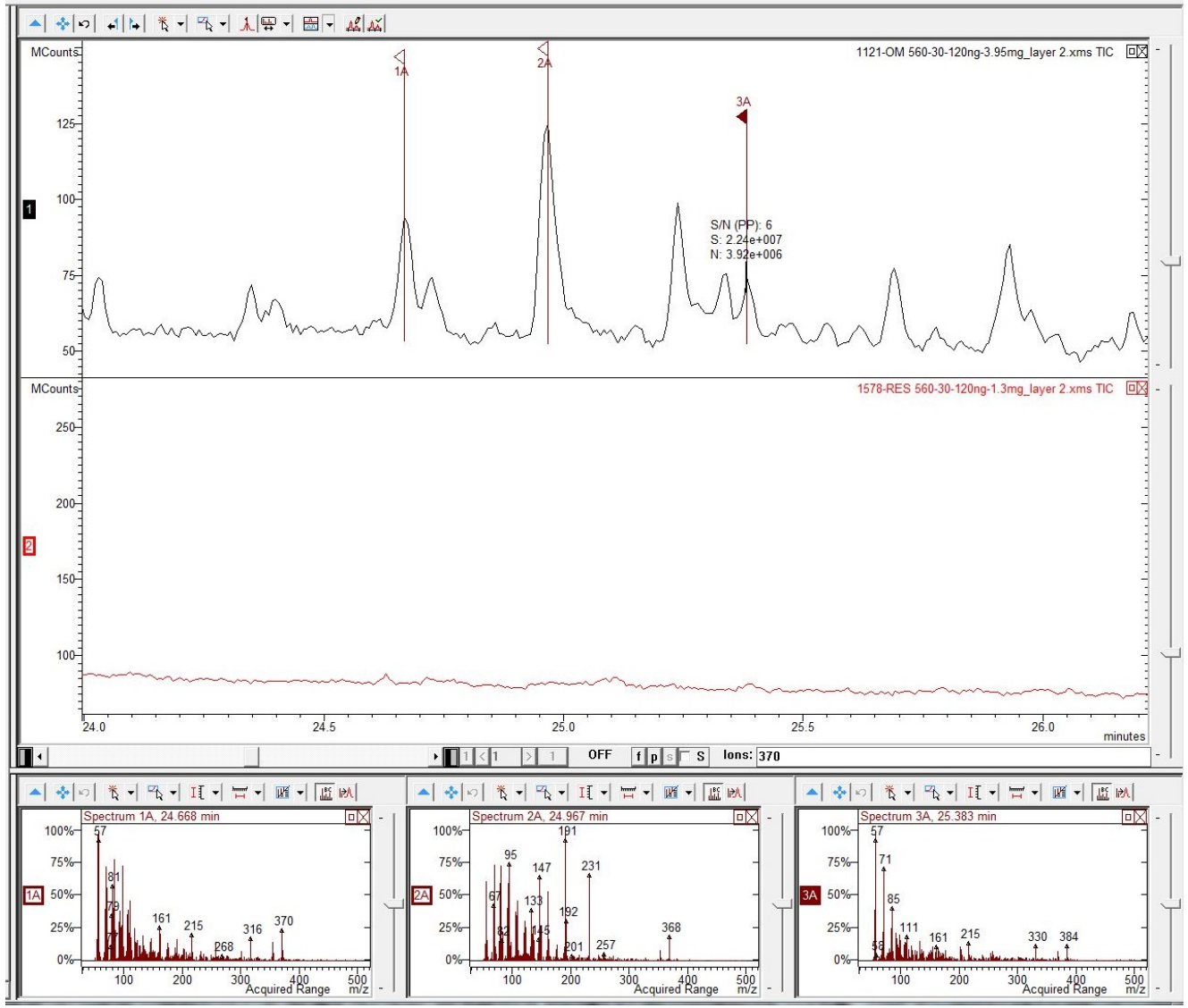

Interactive

comment

Fig. 1. 